\title{
Advances in Nanocomposite Membranes
}

\author{
Pei Sean Goh * and Ahmad Fauzi Ismail
}

Advanced Membrane Technology Research Centre, School of Chemical and Energy Engineering, Universiti Teknologi Malaysia, Johor Bahru 81310, Malaysia; afauzi@utm.my

* Correspondence: peisean@petroleum.utm.my

Citation: Goh, P.S.; Ismail, A.F.

Advances in Nanocomposite

Membranes. Membranes 2021, 11, 158.

https://doi.org/10.3390/

membranes 11030158

Received: 20 February 2021

Accepted: 23 February 2021

Published: 25 February 2021

Publisher's Note: MDPI stays neutral with regard to jurisdictional claims in published maps and institutional affiliations.

Copyright: (C) 2021 by the authors. Licensee MDPI, Basel, Switzerland. This article is an open access article distributed under the terms and conditions of the Creative Commons Attribution (CC BY) license (https:/ / creativecommons.org/licenses/by/ $4.0 /)$.
The design and synthesis of functional nanomaterials have been extensively explored over the last decade, primarily due to their exceptional physico-chemical properties. Various nanomaterials have been used for the development of nanocomposite membranes due to the vast potential in harnessing their compositional and structural advantages. The desired physical and chemical properties endowed by nanomaterials at the molecular level can potentially address the limitations and bottlenecks of conventionally used polymeric and ceramic membranes. The development of nanocomposite membranes involves multidisciplinary strategies that include the innovative design and applications of nanocomposite membranes. The structural and separation properties of membranes can be feasibly tailored and fine-tuned through the introduction of various natural and engineered nanomaterials. When introduced on a surface or within a matrix of membranes, nanomaterials characterized by different dimensional and structural properties render synergetic effects that are unmet by conventionally fabricated membranes.

The Special Issue of "Advances in Nanocomposite Membranes" aims to bring together membrane scientists and engineers to look into multiple aspects associated with the development of state-of-the-art nanocomposite membranes. This Special Issue covers important topics associated to the design and fabrication of nanocomposite membranes, their functionalization and modifications approaches, and the applications of these advanced membranes in niche separation processes, including gas separation, wastewater treatment, and desalination. In total, nine contributions, comprising seven research articles and two reviews, have been successfully compiled in this Special Issue. This collection of research articles and reviews focuses on the synthesis, design, optimization, and applications of advanced nanocomposite membranes, and it also looks into the recent advancements and future trends of this realm.

Metal oxide-based nanomaterials such as titania $\left(\mathrm{TiO}_{2}\right)$ and carbon-based nanomaterials such as graphene oxide (GO) have been extensively used for the development of nanocomposite membranes due to their desired surface hydrophilicity, as well as their textural and structural properties. Based on three types of $\mathrm{TiO}_{2}$-incorporated polyvinylidene fluoride (PVDF) membranes, Teow et al. [1] performed a correlation study between membrane surface morphologies and their fouling behaviors in natural organic mattercontaining surface water. The nanocomposite membrane incorporated with $\mathrm{X}_{500} \mathrm{TiO}_{2}$ exhibited promising anti-fouling and UV-defouling properties, primarily ascribed to its smooth surface enriched with abundant reactive surface groups. By coupling sol-gel and modified phase inversion techniques, Peixoto et al. [2] prepared a cellulose acetate (CA)based nanocomposite incorporated with silica $\left(\mathrm{SiO}_{2}\right)$ and $\mathrm{TiO}_{2}$. In the former nanocomposite membrane, the covalently bound $\mathrm{TiO}_{2}-\mathrm{SiO}_{2}$ was bound to $\mathrm{CA}$ through the interaction between $\mathrm{SiO}_{2}$ and $\mathrm{CA}$, while in the latter, the $\mathrm{TiO}_{2}$ was directly bound to $\mathrm{CA}$. The binding site significantly affected the permeability of the resultant nanocomposites, where the introduction of single $\mathrm{TiO}_{2}$ rendered an improvement of hydraulic permeability by more than two folds when compared to the neat CA membrane. On the other hand, the presence of $\mathrm{SiO}_{2}$ resulted in a neglected change in membrane permeability. 
Alnoor et al. [3] modified the surface of GO and reduced graphene oxide (rGO)deposited polyethersulfone (PES) microfiltration membranes with low-pressure plasma treatment. It was observed that the plasma treatment with a duration of two minutes enhanced the interfacial interaction between the GO or rGO layer and the PES membrane surface. The improved adhesion and stability of the deposited nanomaterial layers were evidenced from their increased potassium chloride ion rejection compared to neat and nanocomposite membranes treated with different plasma treatment conditions. Compared to GO, the deposition of rGO helped to minimize the occurrence of micro- or nano-cracks on the membrane surface, hence further improving the ion rejection to $94 \%$. Roslan et al. [4] proposed a feasible approach to introduce a coating of GO on polysulfone (PSF) hollow fiber membranes. A nanocomposite gas separation membrane consisting of a GO nanosheet, a polyether block amide (Pebax), a polydimethylsiloxane (PDMS) gutter layer, and PSF was developed. Their findings suggested that the introduction of $0.8 \mathrm{wt} \%$ GO into the Pebax selective layer resulted in the best performing multilayered nanocomposite membrane, which exhibited $56.1 \%$ and $20.9 \%$ enhancements in the $\mathrm{CO}_{2} / \mathrm{CH}_{4}$ and $\mathrm{O}_{2} / \mathrm{N}_{2}$ gas pair selectivities, respectively, when compared to the neat membranes.

Nanocomposite membranes incorporated with bi- or tri-metallic nanoparticles were developed by Ndlwana et al. [5] by blending PES with hyperbranched polyethyleneimine (HPEI) supported bi- (Pd@Fe@HPEI) or tri-metallic (Pd@FeAg@HPEI) nanoparticles. The nanocomposite membranes demonstrated increased surface hydrophilicity, textural properties, and pure water permeability. The desired characteristics rendered by the nanomaterials also helped to reduce protein fouling. Quantum dots are semiconductor nanocrystals that show exceptional properties due to quantum mechanics. Gan et al. [6] incorporated lemonderived, carbon quantum dot (CQD)-grafted, silver nanoparticles (Ag) (Ag/CQDs) into a PSF membrane for dye removal. Hydrothermal treatment was employed to carbonize the pulp-free lemon juice into a CQD solution. The findings indicated that the incorporation of $0.5 \mathrm{wt} \% \mathrm{Ag} / \mathrm{CQDs}$ significant improved the pure water permeability and dye rejection by $169 \%$ and $92 \%$, respectively, compared to the neat PSF membranes. The nanocomposite membrane also demonstrated a lower flux decline and irreversible fouling. Tian et al. [7] developed organic-inorganic hybrid membrane by blending various silane compounds with PVDF and hydrochloric acid as a catalyst. It was observed that the silane compounds created artificial ion channels within the nanocomposite membranes, hence improving the cation separation performance of the membranes.

Forward osmosis (FO) has become a new tool for treating various wastewaters and for desalination application. The performance of FO processes greatly depends on the characteristics of the membranes. Suzaimi et al. [8] provided a timely overview on the strategies used for FO thin-film, composite-membrane substrate fabrication and modification. The use of nanomaterials has been highlighted as one of the most versatile and effective strategies in improving substrate layer properties. The roles of novel fabrication and modification in improving water flux, rejection, and antifouling have been thoroughly discussed. A critical consideration of the design of nanocomposite membrane comprises the selection of a well-matched combination of nanomaterials and a polymeric host to cater to intended applications. Goh et al. [9] presented a review to acknowledge the importance of understanding the roles of nanomaterial dimensions in nanocomposite membrane development. The review looked into the selection criteria of nanomaterials from the perspective of their dimensions for the production of high-performance functional nanocomposite membranes for both liquid and gas separation.

The findings and critical discussion from these nine contributions have evidenced the roles and importance of nanocomposite membranes as valuable tools to heighten the performances of various membrane-based separation processes. The flexibility in coupling a wide range of nanomaterials and conventionally used membrane materials offers resultant nanocomposites with high versatility. The authors of these contributions have delved into the important aspects of this topic and addressed many important fundamental questions. The findings have led to a better understanding of material design and the possibilities to 
fill the research gaps. It is envisioned that this Special Issue could illustrate some beneficial guidelines and future trends in the development of more exciting and high-performance nanocomposite membranes.

Author Contributions: P.S.G. and A.F.I. contributed to the planning, drafting, and writing of the editorial. All authors have read and agreed to the published version of the manuscript.

Funding: This research received no external funding.

Acknowledgments: The authors would like to acknowledge the research supports provided by Ministry of Education under Fundamental Research Grant Scheme (FRGS) FRGS/1/2018/STG07/UTM/02/17.

Conflicts of Interest: The authors declare no conflict of interest.

\section{References}

1. Teow, Y.H.; Ooi, B.S.; Ahmad, A.L.; Lim, J.K. Investigation of Anti-fouling and UV-Cleaning Properties of PVDF/TiO 2 MixedMatrix Membrane for Humic Acid Removal. Membranes 2021, 11, 16. [CrossRef] [PubMed]

2. Peixoto, I.; Faria, M.; Gonçalves, M.C. Synthesis and Characterization of Novel Integral Asymmetric Monophasic CelluloseAcetate/Silica/Titania and Cellulose-Acetate/Titania Membranes. Membranes 2020, 10, 195. [CrossRef] [PubMed]

3. Alnoor, O.; Laoui, T.; Ibrahim, A.; Kafiah, F.; Nadhreen, G.; Akhtar, S.; Khan, Z. Graphene Oxide-Based Membranes for Water Purification Applications: Effect of Plasma Treatment on the Adhesion and Stability of the Synthesized Membranes. Membranes 2020, 10, 292. [CrossRef] [PubMed]

4. $\quad$ Roslan, R.A.; Lau, W.J.; Lai, G.S.; Zulhairun, A.K.; Yeong, Y.F.; Ismail, A.F.; Matsuura, T. Impacts of Multilayer Hybrid Coating on PSF Hollow Fiber Membrane for Enhanced Gas Separation. Membranes 2020, 10, 335. [CrossRef] [PubMed]

5. Ndlwana, L.; Sikhwivhilu, K.; Moutloali, R.M.; Ngila, J.C. The Synthesis and Characterization of Novel Bi-/Trimetallic Nanoparticles and Their Nanocomposite Membranes for Envisaged Water Treatment. Membranes 2020, 10, 232. [CrossRef] [PubMed]

6. Gan, J.Y.; Chong, W.C.; Sim, L.C.; Koo, C.H.; Pang, Y.L.; Mahmoudi, E.; Mohammad, A.W. Novel Carbon Quantum Dots/Silver Blended Polysulfone Membrane with Improved Properties and Enhanced Performance in Tartrazine Dye Removal. Membranes 2020, 10, 175. [CrossRef] [PubMed]

7. Tian, Y.; Jin, S.; Zhang, X.; Wang, L.; Lin, Y.; Jin, Y.; Li, L. Organic-Inorganic Artificial Ion Channel Polyvinylidene Fluoride Membranes for Controllable Selectivity Transport of Alkali Metal Cations. Membranes 2020, 10, 174. [CrossRef] [PubMed]

8. Suzaimi, N.D.; Goh, P.S.; Ismail, A.F.; Mamah, S.C.; Malek, N.A.N.N.; Lim, J.W.; Wong, K.C.; Hilal, N. Strategies in Forward Osmosis Membrane Substrate Fabrication and Modification: A Review. Membranes 2020, 10, 332. [CrossRef] [PubMed]

9. Goh, P.S.; Wong, K.C.; Ismail, A.F. Nanocomposite Membranes for Liquid and Gas Separations from the Perspective of Nanostructure Dimensions. Membranes 2020, 10, 297. [CrossRef] [PubMed] 